\title{
Japanese Journal of Mathematics
}

\section{ABBREVIATIONS}

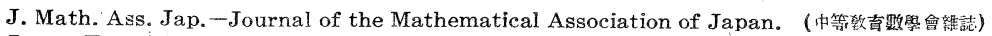
Journ. Fac. Sci. Tokyo.-Journal of the Faculty of Science, Imperial University of Tokyo.

J. Phys. Math. Soc,-Journal of the Physico-Mathematical Society of Japan. (日本数舅物理學會詰)

Mem. Coll. Sci. Kyoto Imp. Univ.-Memoirs of the College of Science, Kyoto Imperial University.

Mem. Ryojun Coll. Engn.-Memoirs of the Ryojun College of Engineering.

Proc. Imp. Acad.-Proceedings of the Imperial Academy.

Proc. Phys.-Math. Soc. Japan.--Proceedings of the Physico-Mathematical Society of Japan.

Sei. Rep. Tôhoku Imp. Univ.-Science Reports of the Tôhoku Imperial University.

Tôhoku Math. Journ.-Tûhoku Mathematical Journal.

Tôkyô But. Z.-Tôkyô Buturigakkô-Zassi. (東京物理學校㩁詰)

J. Fac. Sci. Hokkaidô Imp. Univ.-Journal of the Faculty of Science, Hokkaidô Imperial University.

Sci. Rep. Tokyo Univ. Lit. a. Sci.-Science Reports of the Tokyo University of Literature and Science.

Journ. Sci. Hiroshima Univ.-Journal of Science of the Hiroshima University.

Mem. Fac. Sci. a. Agr. Taihoku Imp. Univ. - Memoirs of the Faculty of Science and Agriculture, Taihoku Imperial University.

Journ. Sci. trop. Agr. Fac. Sci. a. Agr. Taihoku Imp, Univ. - Journal of the Society of Tropical Agriculture, Faculty of Science and Agriculture, Taihoku Imperial University.

\section{ABSTRACTS OF MATHEMATICAL PAPERS PUBLISHED IN JAPAN.}

1. Zahlentheoretische Untersuchungen und Resultate, Alfred MOESSNER. [Tôhoku Math. Jorun , 46 (1940), 234-238.]

2. On axioms af mean transformations and automorphic transformations of abelian groups. Koshichi TOYODA. [Tôhoku Math. Journ., 46(1940), 239-251.] If a set $G$ satisfies the following axioms: (1) for any two elements $a$ and $b$, the product $a \cdot b$ of $G$ determines uniquely one element $c$ in $G$, viz. $a \cdot b=c$. (2) for two given elements $a$ (or $b$ ) and $c$, the equation $a \cdot b=c$ can be uniquely solved by $b$ (or $a$ ) in $G$. (3) for any four elements $a \cdot b \cdot c$ and $d$, we have $(a \cdot b) \cdot(c \cdot d)=(a \cdot c) \cdot(b \cdot d)$. (4) there exists at least one unit element $e$ such that $e \cdot e=e$, then the author calls $G$ an abelian set. If $a \cdot e=e \cdot a^{\prime}$, then $\alpha^{\prime}$ is called the conjugate element of $a$. The fundamental theorems are as follows: (1) For any two elements $x$ any $y, \alpha$ and $b^{\prime}$ are corresponded such that $x=a \cdot e, y=e \cdot b^{\prime}$, and the new product $x * y$ is defined by $x * y=a \cdot b^{\prime}$. Then the abelian set $G$ becomes the abelian group wich respect to the new product $x * y$. (2) A necessary and sufficient condition that a set $G$ forms an abelian set is that the product $x \cdot y$ of $G$ is of the form $x \cdot y=\Gamma_{1}(x) * \Gamma_{2}(y)$ for any elements $x$ and $y$, where $y=\Gamma_{1}(x)$ and $y=\Gamma_{2}(x)$ denote the automorphic transformations of the abelian group $G$ with respect to the product $x * y$, and moreover these operators are mutually permutable, that is, $\Gamma_{1} \Gamma_{2}(x)=\Gamma_{2} \Gamma_{1}(x)$ for any element $x$. The author gives many applications of these theorems,

3. Miscellaneous notes on the history of Chinese mathematics, I. M. FUJIWARA. (in Japanese.) [Tôhoku Math. Journ. 46 (1940), 284-294]. The author reports that three 
mathematical works in the Min-dynasty were found in the cabinet-archive in Tôkyo, and explains their contents. He adds the list of Japanese and Chinese works treating of some half-mathematical and half-divinational problems.

Author.

4. On Taylor Series for which $\lim a_{n+1} / a_{n}=1$. Richard G. COOKE. [Tôhoku Math. Journ, 46 (1940), 319-327.] The first result of this paper is that: Let $f(z)=\sum_{n=0}^{\infty} a_{n} z^{n}$ $=\sum_{n=0}^{\infty} e_{n}\{z /(1-z)\}^{n}$ be a uniform function having $z=1$ as an isolated essential singularity, and no other singularity in the entire plane; also let $e_{n} \geqq 0$ for every $n$. Then $\lim a_{n+1} / a_{n}=1$. The second result is that: If $R_{n}(z)$ is the remainder after the term $\stackrel{n \rightarrow \infty}{a_{n} z^{n}}$ in $\sum_{n=0}^{\infty} a_{n} z^{n}$, and $\lim _{n \rightarrow \infty} a_{n+1} / a_{n}=1$, then $\mathrm{R}_{n}(z) \sim a_{n} z^{n+1} /(1-z)$ uniformly for $|z| \leqq r<1$.

5. Non-vanishing of functions and the related problems. Tatsuo KAWATA. [Tôhoku Math. Journ., $46(1940), 328-339$.$] Suppose that f(x)$ is the Fourier transform of a function $G(u)$ which is real or purely imaginary and belongs to $L_{1}(-\infty, \infty)$ and $L_{2}(-\infty, \infty)$ and vanishes for $u>0$ except in a sequence of non-overlapping intervals $I_{n}=\left(\mu_{n}-1 / 2, \mu_{n}+1 / 2\right)(n=1,2,3, \ldots)$, where $\beta \geqq \frac{\mu_{n+1}}{\mu_{n}}>\alpha>1$ and further suppose that $0<A<G(u) / G(v)<B$ for $\mu_{n}-1 / 2<u, v<\mu_{n}+1 / 2$, where $A, B, \alpha$ and $\beta$ are constants independent of $n$. Let $g(z)=g(x+i y)$ be a function analytic in $c<x<d, 0<y<r$ and continuous in $c<x<d, 0 \leqq y<r$. The author proves that if $f(x)=g(x)$ in some interval in $(c, d)$, then $f(x)=g(x)$ in $(c, d)$. He gives also many applications. For example, if a distribution function $\sigma(x)$ is a step function for $x<0$. with the point spectra $-\mu_{n}\left(\mu_{n}>0\right)$, where $\beta \geqq \mu_{n+1} / \mu_{n} \geqq \alpha>1$, then $\int_{-\infty}^{\infty} e^{i u x} d \sigma(u)$ cannot be a constant different from 1 in any interval.

(M.F.)

6. Continued fractions and matrices. P.M. HUMMEL. [Tôhoku Math. Journ., 46 (1940), 340-359.] This paper is the systematic attempt to apply the theory of matrices with integral elements to continued fractions. Many of the ordinary continued fraction theorems are proved with great simplicity in the new notation, while several theorems proved for binary and ternary continued frantions are extended to the general case, and further new theorems concerning convergence, periodic expansions and the determination of the fundamental unit for all real quadratic fields are proved.

(M.F.)

7. Theorems on Nörlund's method of summation, I, II, Goro HAYASHI and Shinichi IZUMI. [Tôhoku Math. Journ. 47 (1940), 6-13 and 69-73.] The series $\sum_{n=0}^{\infty} a_{n}$ is said to be summable $(N ; Q)$, if $\left(q_{n} s_{0}+q_{n-1} s_{1}+\cdots+q_{0} s_{n}\right) / Q_{n}$ tends to a limit as $n \rightarrow \infty$, where $Q_{n}=q_{0}+q_{1}+\cdots+q_{n}, s_{n}=\sum_{m=0}^{n} a_{m}$. In this paper the relation between $(N ; Q)$ and $(N ; P)$, and relation between $(N ; Q)$ and the ordinary convergence are discussed. (M.F.)

8. Notes on Fourier Series (XI). Inequality theorem in the strong summability, Shinichi IZUMI and Tatsuo KAWATA. [Tôhoku Math, Journ., 47 (1940), 14-17.] For the cosine series $\sum_{k=0}^{\infty} a_{k} \cos k x$ with $a_{k} \geqq 0, \int_{0}^{2 \pi}\left(\sum_{n=1}^{\infty}\left|s_{n}(x)-\sigma_{n}(x)\right| / n\right) d x \geqq c \sum_{k=1}^{\infty} \frac{a_{k}}{k}$ is proved, where $s_{n}(x)=\sum_{k=0}^{n} \alpha_{k} \cos k x, \sigma_{n}(x)=\frac{1}{n+1} \sum_{k=0}^{n} s_{k}(x)$ and $c$ is an absolute constant.

9. On complemented lattices. R.P. DILWORTH. [Tôhoku Math. Journ,, 47 (1940), 18-23.] The author gives negative answer to Husimi's conjecture that a lattice with negation is modular if the chain law holds for every sublattice closed with respective 
to relative negation, and proves a theorem of a similar nature for relatively complemented lattices. He also shows that any complemented, non-modular latice of finite dimensions has a complemented non-modular sublattice of order five. This theorem is the analogue for complemented lattices of the theorem of Dedekind that any non-modular lattice contains a non-modular sublattice of order five. As an application, he gives a new proof of the theorem due to G. Birkhoff and M. Ward that a lactice of finite dimensions is a Boolean algebra if and only if every element has a unique complement.

(M.F.)

10. Über die isomorphe Darstellung der endlichen Gruppe. Masatada TAZAWA. [Tôhoku Math. Journ., 47 (1940), 87-93.] The object of this paper is to solve the following problem: For a finite group $G$, when is there an isomorphic representation of $G$ which is decomposable into $k$ irreducible factors, for a given finite group? For the case $k=1$, Shoda has obtained the perfect solution. This paper gives the perfect solution for the general case.

11. Miscellaneous notes on the history of Wazan, III. (in Japanese.) M. FUJIWARA. [Tôhoku Math. Journ., 46 (1940), 295-308.] This paper treats the following subjects: (1) "Jinkoki" in Kan-ei 18-th year and problem of determination of the volume of sphere, (2) succesive approximation in "Sampo Hutudankai", (3) recurring formulas treated by Nakane Genkei. Author.

12. Miscellaneous notes on the history of Chinese mathematics, II. (in Japanese.) M. FU. JIWARA. [Tôhoku Math. Journ., 47 (1940), 35-48] This paper explains minutely the approximation method to solve numerical algebraic equations in "Yôki-sampô" and "Sampô Tôsô", and their relations to Japanese mathematics.

Author.

13. Miscellaneous notes on the history of Wazan, IV. (in Japanese.) M. FUJIWARA. [Tôhoku Math. Journ., 47(1940), 94-57.)] The author points out that Takakazu Seki, receiving hint for the methods to solve approximately numerical algebraic equations from "Yôki-sampo" succeeded to establish perfectly the so-called Horner's method.

Author.

14. Die projektive Differentialgeometrie als eine Verallgemeinerung der N.E. Differentialgeometrie II. Kurventheorie im Raume. Saburo SATO. [Tôhoku Math. Journ., 46 (1940), 181-233.] Der Verfasser benutzt nach Takasu instantane absolute Quadrik (oder kürzer absolute Quadrik)

$$
a_{i k} x^{i} x^{k}=k^{2}(\sigma),
$$

wobei $a_{i y_{i}}$, Funktionen vom Parameter des Kurvenpunktes sind, und behandelt projektive Differentialgeometrie der Raumkurven als eine Verallgemeinerung der N.E. Geometrie, wobei die absolute Quadrik als fest angenommen ist. Dadurch gibt der Verfasser mehrere geometrische Deutungen der projektiven Differentialinvarianten. Zum Schluss gibt der Verfasser die Beziehungen seiner Theorie zu den Theorien von Fubinischen Projektivdefferentialgeometrie und von Blaschkeschen Afflndifferentialgeometrie von den Raumkurven.

15. A continuous group of contact transformations containing the generalized pedal transformation. J. M. FELD. [Tôhoku Math. Journ. 46 (1940), 252-260.] If the pedal transformation $T$ ' on a plane is repeated, the result is contact transformation $T \cdot T=T^{2}$. Similarly $T^{3}, T^{4}, \ldots$ are also contact transformations and likewise the inverse of $T$, namely $T^{-1}$ and its successive iterates $T^{-2}, T^{-3}, \ldots$ are contact transformations. This 
discrete family of $\infty^{1}$ transformations $T^{n}$ ( $n$ an integer) does not constitute a continuous group. Lie succeeded, however, in imbedding these transformations in a continuous oneparameter group. To do so he expressed the pedeal transformation in polar coordinates. Letting the pole of the polar system serve as pole of the pedal transformation, $T$ can be expressed in the form

$$
r_{1}=r \sin \tau, \phi_{1}=\phi+\frac{\pi}{2}-\tau, \tau_{1}=\tau
$$

where the line elements are represented by $(r, \phi, \tau)\left(r_{1}, \phi_{1}, \tau_{1}\right)$. The $n$-th iterate $T n$ is thus found to be $\quad r_{1}=r(\sin \tau)^{n}, \phi_{1}=\phi+n\left(\frac{\pi}{2}-\tau\right), \tau_{1}=\tau$.

By allowing $\boldsymbol{n}$ to have all real values, (1) represents a one-parameter continuous group of contact transformations containing the pedal transformation and its iterates.

In this paper Lie's results are generalized in two directions. First, a three parameter continous group of contact transformations is constructed in which all the iterates of a projective generalization of the pedal transformation are contained and also the iterates of a projective generalization of $\infty^{1}$ skew pedal transformations. By a skew pedal curve of a given curve $C$ with respect to the pole $O$ is meant the locus of the points $P$ on the tangents of $C$ such that $O P$ and the tangents intersect at a constant angle different from $\frac{\pi}{2}$. Secondly these groups are generalized to $n$-space. Furthermore some applications to the geometry of triangular symmetric curves and also to $W$ curves are indicated.

16. Some simple applications of Green's theorem for compact Riemann spaces. T.Y. THOMAS. [Tôhoku Math. Journ., 47 (1940), 261-266.] In this note the author considers an $\boldsymbol{n}(\geqq 2)$ dimensional oriented compact Riemann space which we denote by $R$. In the paragraphs 1 and 2 it is assumed that $R$ is of class $C^{1}$ and in the remainder of this note it is assumed that $R$ is of class $C^{2}$.

1. If $\lambda$. be a vector of class $C^{1}$ over $R$, then

$$
\int d i v \lambda d v=0 \quad \text { (Green's theorem) }
$$

2. Consider $\Delta \varphi=\lambda$ ? where $\lambda=$ const.

It follows that $\Delta\left(\varphi^{2}\right)=2 \lambda \cdot \mathscr{q}^{2}+2 S(\mathrm{grad} \rho)$ and we have Theorem 1 . If $\lambda>0$, the conly solution of (2) over Riemann space $R$ is given by $\varphi=0$ and if $\lambda=0$, the only solution of this equation (2) is $p=$ const.

If $T$ is any tensor over $R$ and $T_{\mu \ldots . \beta}^{\alpha \ldots \beta}$ denote its components, put

$$
\Delta T_{\mu \ldots \nu}^{\alpha \cdots \cdots}=g^{\sigma \nu} T_{\mu \cdots, \gamma, \sigma, \tau}^{\alpha \cdots \cdots,}
$$

where the right member involves the second covariant.

Consider $\Delta \psi_{\alpha}=\lambda i_{\alpha}$,

where $\lambda$ denotes a cretain constant.

Theorem III. If $\lambda>0$, the only vector field $\psi$ satisfying (3) over $R$ is the null-vector field and if $\lambda=0$ any vector field $\psi$ which satisfies (3) over $R$ possesses a covariant derivative which vanishes identically.

We finally arrive at 
Theorem VIII. Two oriented compact Riemann spaces of class $C^{m}(m \geqq 2)$ and dimensionality $n \geqq 2$ having zero Gaussian curvature and equivalent conformally have fundamental metric tensors which are not essentially different.

17. On line congruence, IV. Kusuo TAKEDA. [Tôhoku Math. Journ., 46 (1940), 267-283.] In this note the author proves at first that there exist $\infty^{2}$ osculatihg cubic complexes $\mathbb{E}_{3}$ having contact of the sixth order with the given line ccngruence $K$ along the line $p$ and each of them is composed of two complexes; one of them is the osculating quadratic complex $\mathbb{S}_{2}$ while the other is a linear complex having in $R_{5}$ the tangential plane of $K$ at $p$ as its singular plane.

There exist seven directions of the ruled surface of $K$ having the contact of the seventh order with $\mathfrak{S}_{3}$ along $p$. Then the author gives the necessary and sufficient condition in order that a ruled surface of $K$ has the coincident flecnote tangents and gives the differential equation for it. Further the author discusses the projective geometric properties of osculating linear congruence of some associated ruled surface of the line congruence and so forth.

18. General differential geometries with coordinate interspace inner product. A.D. MICHAL and D. H. HYERS. [Tôhoku Math. Journ., 46 (1940), 309-318.] In the general differential geometries with Banach coordinates and with a linear connection, there is a certain amount of arbitrariness in the choice of a definition for a covariant vector. A natural definition that employs a Banach coordinate space with an independently postulated bilinear inner product has already been given in the authors' provious notes. In this note the authors consider briefly two Banach spaces $B_{1}$ and $B_{2}$ with a postulated interspace inner product $(z, L)$ satisfying the properties

$$
\begin{aligned}
& \text { i. }(x, L) \text { is bilinear on } B_{1}, B_{2} \text { to real numbers, } \\
& \text { ii. If }(x, L)=0 \text { for all } x \varepsilon B_{1} \text {, then } L=0, \\
& \text { iii. }(x, L)=0 \text { for all } L \varepsilon B_{2} \text {, then } x=0,
\end{aligned}
$$

where the notion of symmetry in this interspace inner product has no meaning when $B_{1}, B_{2}$ are distinct. In $\xi_{4}$ the authors use such an interspace inner product to generalize the natural definition of covariant vectors referred to above. In this note the authors discuss the elementary properties of adjoint transformations and their differentiability in a parameter.

19. Einige Bemerkungen $z u$ den Eilinien. Tadahiko KUBOTA. [Tôhoku Math. Journ., 47 (1940), 1 5.] Proofs of the two following theorems :

Take two fixed points $A, B$ on the oval $\&$. Sappose that the oval $E$ has the property :

If we draw two arbitrary parallel chords $A A_{1}, B B_{1}$ of the oval \& passing through $A, B$ and the two arbitrary parallel chords $A_{1} A_{2}, B_{1} B_{2}$ of the oval \& passing through $A_{1}$, $B_{1}$, then the chords $A B_{2}, A_{2} B$ are parallel. Then the oval \& must either an ellipse or a central curve.

If the relative circle of curvature of an are $A C B$ be monotoneously increasing from $A$ to $B$, then the relative-circular are $R$ passing through $A, B$ and touching the given curvilinear arc $A C B$ at $A$, cuts the chord $A B$ at $B$ in the greater angle than the angle at $B$ which the are $A C B$ makes with the chord $A B$, under the assumption that the arc $A C B$ makes with the chord $A B$ an oval. (This is a generalization of Vogt's theorem.) Author.

20. Ein Satz über Eilinien. Tadahiko KUBOTA. [Tôhoku Math Journ., 47 (1940), 76-98.] In this note the author has proved the following theorem: Die Eilinie \& besize 
überall der Krümmungskreis, der sich entlang der Eilinie c stetig ändert. Der grösste und der kleinste Krümmungsraduis der Eilinie $\mathbb{E}$ seien bzw. mit $r_{A}, r_{B}$ bezeichnet, wobei $A, B$ ihre Brrührungspunkte bedeuten. Konstruiert man nun einen Kreis mit dem Radius $r$, der diese Eilinie $E$ mindestens in drei verschiedenen oder koinzidenten Punkten schneidet, dann ist

$$
r_{B} \leqq r \leqq r_{\mathrm{A}} \cdot
$$

This theorem is essentially the same as Brunn's theorem which was kindly remarked to me by Mr. Minoda. The proof is quite different fróm Brunn's proof.

Author.

21. Ein neuer Beweis des Vogtschen Satzes. S KATSUURA. [Tôhoku Math Journ., 47 (1940), 94-95.] A very simple geometric proof of Vogt's theorem concerning osculating circles of the curvilinear arc with monotoneously increasing radius of curvature.

(T.K.)

22. On some osculating figures of a plane curve, and on sections of a surface by planes passing through a fixed tangent. Jusaku MAEDA. [Sci. Rep. Tôhoku Imp. Uiv. Ser. 1, $28(1940), 163-203$.$] The subject-matters are: On a certain circular cubic as-$ sociated with a point on a curve; double spirals; isogonal trajectories of confocal conics; isogonal trajeciories of confocal Cassinian ovals; hypocycloids and epicycloids; cycloids; tractrices and catenaries; on sections of a surface by planes passing through a fixed tangent.

Author.

23. On the theory of curves in Euclidean three-space. Jusaku MAEDA. [Sci. Rep. Tôhoku Imp. Univ. Ser. 1. 28 (1940), 319-333.] The author proves, among others, the following theorems.

If the torsion of a space curve is constant, then its affine binormal at every point. on it lies in its rectifying plane at that point; and conversely.

Let $(M)$ be a given space curve and $M$ a point on it; let $l$ be a given straight line passing through the point $M$ and not contained in the osculating plane $\pi$ of the curve $(M)$ at the point $M$ : and let the projection of the given curve $(M)$ in the direction of the straight line $l$ on the osculating plane $\pi$ be denoted by $(M *)$; if the osculating conic at $M$ of $\left(M^{*}\right)$ is an equilateral hyperbola, then the straight line $l$ describes a quadric cone.

Author.

24. A remark concerning plane curves. Jusaku MAEDA. [Sci. Rep. Tôhoku Imp. Univ. Ser. 1, 28 (1940), 334-349.] The locus of the foci of conics having at least fourpoinc contact with a given curve at a point is a circular cubic. The author establishes, among others, many properties of this circular cubic.

Author.

25. On the theory of curves in affine space. Jusaku MAEDA. [Sci. Rep. Tôhoku Imp. Univ. Ser. 1, 28 (1940), 350-369.] The subject-matters are: the affine analogues of the curves of Cesaro; geometrical meanings of the affine binormal of Winternitz; the complex pints of the affine principal normal surface and the affine binormal surface of a space curve; the flecnodes of the affine binormal surface; the osculating quadric of the affine binormal surface. The author proves, among others, the following theorem, which gives a characteristic property of curves of constant affine curvature. If the affine curvature of a space curve is constant and not equal to zero, then the given curve is a flecnode curve on its affine binormal surface; and conversely. Author. 
26. Sur la podaire d'une hypersurface dans l'espace euclidien à n dimensions. Jusaku MAEDA. [Tôhoku Math. Journ., 46 (1940), 306-384.] Soient (MI) une hypersurface dans $E_{n}, M$ un point de $(M),(\bar{M})$ la podaire de $(M)$ par rapport à un point quelconque, qui n'est pas sur l'hyperplan tangent ì $(M)$ en $M$, éc $\overline{M I}$ le point correspondant au point $M$ par cette transformation podaire; alors les deux directions sur $(M)$, issues de $M$, qui correspondent, par cette transformation podaire, à deux directions $\operatorname{sur}(\bar{M}$, issues de $\bar{M}$, sont orthogonales et conjuguées entre elle.

Author.

27. Une propriété caractéristique des courbes gauches dont les courbures projectives sont constantes. Jusaku MAEDA. [Tôhoku Math Journ., 47(1940), 74-76.] Si une ligne droite, qui passe par un point mobile $M$ d'une courbe gauche $(M)$, autre qu'une cubique gauche, et qui est fixe projectivement par rapport au tétraèdre normal ou au tétraèdre fondamental, quand le point $M$ se meut le long de $(M)$, une surface développable, alors la courbure projective de $(M)$ est constante. Réciproquement, pour une courbe gauche $(M)$ à courbure projective constante, il y a une infinité de lignes droites, passant par un point mobile $M$ de $(M)$ et fixes projectivement par rapport au tétraèdre normal ou au tétraèdre fondamental de $(M)$, telles que chacune d'elles engendre une surface développable quand le point $M$ se meut le long de $(M)$; et la totalité de ces droites forme un cône du second ordre touchant le plan osculateur de $(M)$ à $M$ le long de la tangentc de $(M)$ ì $M$.

Author.

28. On the section of a surface by a variable plane passing through a fixed tangent line. Jusaku MAEDA. [Tôhoku Math Journ., 47 (1940), 58-68.] If we cut a given surface by a moving plane passing through a fixed tangent line, which is not asymptotic, at a point $O$ of the surface and construct the osculating parabola, the osculating equilateral hyperbola and the osculating equiangular spiral of the curve of section at $O$, then the directrix of the parabola describes a quadric, the focus of the same parabola describes a circle, the equilateral hyperbala describes a quadric, the centre of the same hyperbola describes a circle, and the pole of the equiangular spiral describes a circle. Author.

29. On systems of rectangular hyperboloids and orthogonal hyperboloids associated with a point of a surface. Jusaku MAEDA. [Tôhoku Math. Journ., 47 (1940), 24-34.] The locus of the centre of any orthogonal hyperboloid having a contact of the second order with a given surface at a non-parabolic point is a sphere, which is the reflexion of the middle sphere of the surface at the point with respect to the tangent of the surface at the point.

The locus of the centre of any rectangular hyperboloid having a contact of the second order with a surface at a non-parabolic point is a quadric.

If the locus of the pole of a plane with respect to any orthogonal hyperboloid having a contact of the sec nd order with a given surface at a non-parabolic point is an orthogonal hyperboloid, then the locus of the centre of such an orthogonal hyperboloid obtained in this manner is the middle sphere of the surface at the point.

If the envelope of tht polar plane of a point with respect to any orthogonal hyperboloid hav.ng a contact of the second order with a given surface at a non-parabolic point is an ortbogonal hyperboloid, then the locus of the centre of such an orthogonal hyperboloid obtained in this manner is the central sphere of the given surface at the point.

Author.

30. On the problems of equivalence of plane curves in the Lie's higher circle geometry and of minimal curves in the conformal geometry. S. SASAKI and T. SUGURI. [Tôhoku. Math. Journ., 4.7 (1940), 77-£6.] One of the most fundamental but not yet completely 
solved problem in the Lie's higher circle geometry (Möbius' sphere geometry with the signature ++-) is " To obtgin the necessary and sufficient conditions that two plane curves (two minimal curves) $c$ and $c^{\prime}$ are transformable into each other by a Lie's circle transformation (Möbius' conformal transformation)". We have solved this problem in seeking for the natural arc lengths and curvatures of curves by the method of repères mobiles. The only exceptional curves corresponding to straight lines and conics in the projective planes are those which admit $\infty^{3}$ Lie's (Möbius') transformations transforming the curves into themselves (and minimal lines also in the case of Möbius' geometry).

Authors.

31. On the theory of surfaces in a curved conformal space. S. SASAKI. [Sci. Rep. Tôhoku Imp. Univ. Ser. I, 28 (1940), 261-281.] In this paper the author deals with the basic apparatus for the theory of hypersurfaces in a curved conformal space in the sense of Princeton's school (which may be regarded as the space endowed with the normal conformal connexion in the sense of E. Cartan) in a manner of aouble tensor analysis with respect to the enveloping space and the hypersurface itself. On account of the fact that the connexion on the hypersurface induced from the enveloping space is also the normal conformal connexion, the basic formulas (Gauss' and Weingarten's equations and the conditions of integrability) are somewhat complicated. Author.

32. Geometry of the conformal connexion. S. SASAKI. [Sei. Rep. Tôhoku Imp. Univ. Ser. I. 29 (1940), 219-267.] The geometry of the conformal connexion is largely concerned with the conformal geometry of Riemannian spaces and the Möbius' conformal geometry. But the second does not contain the third as its special case. First the paper explains this fact and then studies those spaces with normal conformal connexions admitting groups of conformal point transformations using the complete set of conformal differential invariants (Chap. I). In Chap. II the paper treats generalized circles obtaining their differential equations by a simple method. Then introducing the (induced) conformal connexions, which are not normal in general, on subspaces from the enveloping space, there are considered the geometry of subspaces in a conformally connected space. The basic formulas are simpler than those of the former paper (Chap. III). In the last chapter some geometrical properties of hypersurfaces are discussed and there are studied the relations of our geometry of hypersurfaces and the G. Thomsen's theory of surfaces in the Möbius' geometry.

Author.

33. On a remarkable property of umbilical hypersurfaces in the geometry of the normal conformal connexion S. SASAKI. [Sci. Rep. Tồhoku. Imp. Univ. Ser. I. 29(1940), 412422.] In the theory of hypersurfaces in the geometry of the normal conformal connexion we meet an important scalar $\lambda$. and two (induced and intrinsic = normal) conformal connexions for the hypersurface induced from the enveloping space. In this paper the author studies those hypersurfaces for which $\lambda$ 's defined by the intrinsic connexions vanish identically and those hypersurfaces for which the induced connexions coincide with the intrinsic connexions. The former are umbilical hypersurfaces and the latter constitute a subclass of them. But if the connexion of the space is flat, any umbilical hypersurfaces belong to the latter class. Accordingly we may regard this fact as a differential geometrical characterization of hyperspheres in Euclidean geometry.

Author.

31. Über die Struktur der l-Klassengruppe zyklischer Zahlkörpers vom Primzahlgrad I. Eizi INABA. [Journ. Fac. Sci., Tokyo Sec II. 4(1940), 61-115.] Es handelt sich haupisächlich um einen tiefen Zusammenhang, der zwischen der Struktur der-Klassen- 
gruppe des relativzyklischen Zahlkörpers vom Primzahlgrad $l$ und einer Reihe von gewissen Diophantischen Gleichungen besteht. Dafür wird nämlich konstruiert eine Gruppe der Ideale (oder Zahlen) im Grundkörper, welche sich durch Diskriminantenprimteiler und Lösungen der Gleichungen erzeugen lässt. Jene Gruppe spiegelt die Struktur der $l$-Klassengruppe des Oberkörpers vollständig ab und ermöglicht diese durch ein endliches Rechen verfahren zu bestimmen. Die vorliegende Betrachtungsweise gestaitet auch viele schon bekannte Tatsachen auf neuem Wege wieder herzuleiten, insbesondere die Resultate von Redei, Reichardí, Moriya. Zum Schluss wird noch des Lösbarkeitskriterium der verallgemeinerten Lagrangeschen Gleichung hinzugefügt. Author.

35. Ideals in a Boolean algebra with transfinite chain condition. Fumitomo MAEDA. [Journ. Sci. Hirosima Univ. A $\mathbf{1 0}(1940), 7-36$.$] Let a be an s-ideal in a generalized$ Boolean algebra $L$. When $L / \mathfrak{a}$ satisfies the $z$-chain condition, a is called a basic $s$-ideal of the s-chain condition. Then the class $\mathfrak{B}_{5}$ of all basic $:$-ideals of the 5 -chain condition in $L$ is a generalized $\leq-B o o l e a n$ algebra, and the class of all dual $\leqslant$-ideals in $\mathfrak{P}_{z}$ is a continuous Boolean algebra. The author applies this result to the class of all measure functions defined in an $:$-Boolean algebra and to the spectral theory of the complete complex Euclidean space.

Author.

36. Über die Noetherschen fünf Axiome in kommutativen Ringen. Keizi KUBO. [Journ. Sci. Hirosima Univ. Ser. A. 10 (1940), 77-84.] It is well known that, if the five conditions of Noether are satisfied in a commutative ring $\Re$, then every ideal of $\Re$ can be represented as a unique product of a finite number of prime ideals. But these conditions are not necessary. The paper is devoted to this problem and the main result is the following: In order that every ideal in a commutative ring $\Re$ can be represented as a unique product of a finite number of prime ideals, it is necessary and sufficient that (1) the descending chain condition for ideals holds, (2) there exists the unit element, (3) the redical of $\mathfrak{n}$ is prime and (4) there is no ideal between $\mathfrak{p}$ and $\mathfrak{p}^{2}$, if $\mathfrak{p}$ is a prime ideal.

37. Über die Produktzerlegung der Hauptideale. III. Shinziro MORI. [Journ. Sci. Hirosima Univ. Ser. A. 10 (1940), 85-94.] The author proves the following theorem and its converse: Let $\Re$ be a commutative ring with unit such that every principal ideal of $\Re$ is a power product of a finite number of prime ideals, then $\Re$ is the direct sum $\Re=\Re_{1}+\Re_{2}+\cdots+\Re_{n}(n<\infty)$ where $\Re_{i}$ is (1) a primary ring with unit, in which the unique prime ideal is a principal ideal, or (2) an integral domain, in which every chain of idealquotients $(a): \mathfrak{a}_{1}<(a): \mathfrak{a}_{2}<\cdots$ ends in finite and moreover every highest prime ideal of any principal ideal is reversible.

Author.

38. Geometry of parallel displacement making a volume invariant. Minoru URABE. [Journ. Sci. Hirosima Univ. Ser. A. 10 (1940), 95-108.] In a $n$-dimensional metric space $X_{n}$, the author defines the volume $V$ spanned by $m$ vectors $v_{1}^{2}, v_{2}^{2}, \ldots v^{2}$ as follows :

$$
V=v \underset{1}{a} v_{2}^{[1} v^{2} \ldots v_{m}^{m} e_{m+1}^{m+1} \ldots e_{n}^{n]}
$$

where $e_{m+1}^{\nu}, e_{m+2}^{\nu}, \ldots e_{n}^{\nu}$ are unit vectors orthogonal to $v_{1}^{\nu}, v_{2}^{\nu}, \ldots v_{m}^{\nu}$, and perpendicular to each cther, and $a^{n}=$ det. $\left|\alpha_{\lambda \mu}\right|, a_{\lambda \mu}$ being any given tensor. Then, if $m<n$, the coefficients of connection, which give the parallel displacement making this $m$-dimensional volume invariant, are given by 


$$
\Gamma_{\lambda \mu}^{\nu}=\left\{\lambda_{\lambda \mu}^{\nu}\right\}-\frac{1}{m}\left(Q_{\lambda} A_{\mu}^{\nu}+Q_{\mu} A_{\lambda}^{\nu}-g_{\lambda \mu} Q_{\nu}\right)-S_{\cdot \mu \lambda}^{\nu}-S_{\lambda_{\lambda \mu}}^{\nu}+S_{\lambda \mu}^{\nu},
$$

where $Q_{\nu}=\partial_{\nu} \log \sqrt{\frac{g}{a}}$,

$$
\begin{aligned}
& S_{\lambda \mu}{ }^{\nu}=\Gamma_{[\lambda \mu]}^{\nu}, \\
& g=\text { det. }\left|g_{\lambda \mu}\right|, g_{\lambda \mu} \text { being fundamental tensor. }
\end{aligned}
$$

When the space $X_{n}$ is affine, i.e. $S_{\nu \mu}^{* \nu}=0$,

$$
\Gamma_{\lambda \mu}^{\nu}=\left\{\nu_{\nu \mu}^{\nu}\right\}-\frac{1}{\dot{m}}\left(Q_{\nu} A_{\mu}^{\nu}+Q_{\mu} A_{\lambda}^{\nu}-g_{\lambda \mu} Q^{\nu}\right) \text {. }
$$

This shows that the space has Weyl's connection and is conformal to Riemannian, since $Q_{\nu}$ is a gradient vector. When the field $a_{\lambda \cdot \ell}$, by which the volume is defined, is given as a parallel tensor field, the space becomes Riemannian, provided that the space $X_{n}$ is affine. If $m=n$, we have "inhaltstreu" parallel displacement.

Next, he has investigated the coudition that this parallel displacement may be induced by a group, and found that Blaschke's affine geometry is a special case of the geometry of that group. Specially in the case of constant curvature this group becomes the rotations and dilatations, when the number of parameters of the group is not less than $\frac{n(n+1)}{2}$.

Author.

39. Allgemeine Z.P.I. Ringe. Shinziro MORI. [Journ. Sci. Hirosima Univ. Ser. A. 10 (1940), 117-136.] A commutative ring, in which every ideal is expressible in a product of a finite number of prime ideals (not necessary unique), is called a general Z.P.I. ring. In recent years several interesting contributions to the structure of the particular Z.P.I. rings have appeared. In the present paper the author discusses this problem in the most general case and obtains the following results:

I. In order that a commutative ring $\Re$ be a general Z.P.I. ring, ic is necessary and sufficient that (a) $\mathfrak{n}$ satisfies the ascending chain condition for ideals, (b) there is no ideal between $\mathfrak{p}$ and $\mathfrak{p p}^{\prime}$, if $\mathfrak{p}$ and $\mathfrak{p}^{\prime}$ are prime maximal ideals and (c) there is no ideal between $\Re$ and $\Re^{2}$.

II. In order that a commutative ring $\Re$ with unit be a general Z.P.I. $\operatorname{ring}$, it is necessary and sufficient, that $\Re$ satisfies the ascending chain condition for ideals and there is no ideal between $\mathfrak{a}$ and $\mathfrak{a}^{2}$, if $\mathfrak{a}$ is a maximal ideal in $\mathfrak{H}$.

Author.

40. Partially ordered linear spaces. Fumitomo MAEDA. [Journ. Sci. Hirosima Univ. A 10 (1940), 137-150.] Author finds a generalized ₹-Boolean algebra in the partially ordered linear space $Y$, and using the same method as the theory of set functions he obtains the incegral representation of the elements in $Y$ which are obtained by Freudenthal in a different way.

Author.

41. Hypermaximalität normaler Operatoren. Hidegorô NAKANO. [Proc. Phys-Math. Soc. Japan, Ser. 3, 22 (1940), 259-264.] In der früheren Abhandlung (Zur Eigenwerththeorie normaler Operatoren, Proc. Phys.-Math. Soc. Japan, 21 (1939), 315-339) hat Verfasser Spektralcheorie normaler Operatoren in neuer Art bohandelt. Hier ist gezeigt, dass bisherige Spektraltheorie selbstadjungierter Operatoren oder normaler Operatoren in unserer Theorie enthalten ist.

Author.

42. Theorems on "schlicht" functions, IV. Kenzo JOH: [Proc. Phys.-Math. Soc. Japan, Ser. 3, 22 (1940), 329 343.] The main theorem proved in this paper is as follows: 
Let $w=F(z)$ be the functions under the following condition; (i) $F(z)$ is regular for $|z|<R$ (ii) $F(0)=0$ (iii) $F(z) \neq 1$ for $|z|<R$ (iv) $w=F(z)$ reprensents the circle $|z|<R p$-valenlty to the domain $G_{p}$ on the Riemann surface of $\sqrt[p]{w-1}$. And let $\delta$ be the Lebesgue measure of the set of points on $|z|=R$ for which the radial limiting value $\lim _{z: R}|F(z)| \geqq F_{i} 2 p$, then $\delta<8$. And for any rabitrary given small number $\varepsilon$, there

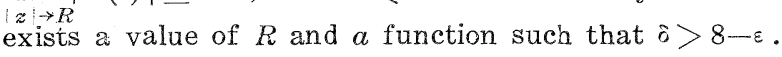

(M.T.)

43. Sur l'arête de Green d'une surface plongée dans un espace à connexion projective. Jôyô KANITANI. [Prcc. Phys. - Math. Soc. Japan, Ser. 3, 22 (1940), 343-358.] En genéralisant la quadrique de Lie d'une surface dans un espace projectif pour une surface plongée dans un espace à connexion projective nous obtenons deux quadriques qui ne sont pas en coincidence en général. Au moyen de ces quadriques nous definissons deux droites qui sont les réciproques l'une de l'autre. Nous pouvons alors definir l'arête de Green par la methode de M.G. Green, et la plupart des propriètes de cette arête peuvent être aussi généralisées.

(From the author's introduction).

44. Doppelberïhrungslehre der Kurven zweiter Ordnung, II. Sadataka MIYAZAKI. [Proc. Phys.-Math. Scc. Japan. Ser. 3, 22 (1940), 399 423.] This is the continuation of the author's former papar. (Japanese Journ. Math., 16 (1939), 135-147).

45. On the tangents of the reciprocal derivative in $E_{4}$. Matsuji TSUBOKO. [Prcc. Phys.-Math. Soc. Japan, Ser. 3, 22 (1940), 509 518.] Let $x$ be a moving point on a curve $\Gamma_{x}$ in the four dimensional projective space $E_{4}, y$ be a point in the sclid osculating $\Gamma_{x}$ at $x, \Gamma_{y}$ be a curve described by the point $y$, as $x$ moves along $\Gamma_{x}$ and $S$ be the ruled hypersurface generated by the plane $\tau_{1}$, determined by $y$ and the tangent at $x$ of $\Gamma_{x}$. When the plane osculating $\Gamma_{y}$ at $y$ passes through $x$, the curve $\Gamma_{y}$ is called the reciprocal derivative curve of $\Gamma_{x}$. The author has investigated some properties of such curves in the former papar (Proc. Phys.-Math.-Soc., Ser. 3, 21 (1939), 298-311). The author studies in this paper another characterisation of the tangents of $\Gamma_{y}$ by making use of line coordinates and discusses the relations between the locus of those tangents and the ruled hypersurface $\mathrm{S}$.

46. Sur la connexion de Weyl-Hlavatý et ses applications à la géometrie conforme. Kentaro YANO. [Proc. Phys.-Math. Soc. Ser 3, 22 (1940), 595-621.] It has been shown that the linear connection $\Pi_{\mu \lambda}^{\lambda}=\left\{\lambda_{\mu \nu}^{\lambda}\right\}+\hat{c}_{\mu}^{\lambda} p_{\nu}+\delta_{\nu}^{\lambda} p_{\mu}-g^{\lambda \alpha} p_{\alpha} g_{\mu \nu}$ of Weyl-Hlavatý can be used to study the conformal geometry of Riemann sfaces. By an elimination method, the author obtains the conformal curvature tensor of Weyl, the differential equations of conformal circles and the projective parameter on it. Moreover, he obtains the Frenet formulae for the curves in the conformal geometry of Riemanninn spaces.

Auchor.

47. On some properties of sabspaces in a conformally connected marifold. Yosio MUTÔ. [Proc. Phys.-Math. Sac., Ser. 3, 22 (1940), 621-636.] The purpose of this paper is to study some of the properties of subspaces other than hypersurfaces in a conformally connected manifold.

(M.T.)

48. On uniform spaces and the dimension of compact spaces. Kiiti MORITA. [Proc. Phys.-Math. Sce. Japan, Ser. 3, 22 (1949), 969-977.] Recently A. Weil has introduced a new notion of uniform spaces as a generalisation of that of metric spaces. In this papar the author first $(\xi 1)$ discusses some thorems in the dimension of bicompact spaces from the standpoint of uniform spaces. After Weil he considers a finite covering 
by open sets $G$ of a space $S$ and corresponding tis it a subset $V$ of $S \times S$, defined by $V_{\mathscr{C}}=\sum_{i=1}^{m}\left(G_{i} \times G_{i}\right)$, where $G=\left\{G_{1}, \ldots, G_{m}\right\}$. If the family $\left\{V_{(S)}\right\}$ formed by all $V_{\text {BS }}$ (S extending over all finite coverings of $S$ by open sets) defines a uniform topology that is equivalent to the topology which is given in $S$ beforehand, it will be said that a space $S$ has the property $(E)$.

In $\& 2$ the author determines spaces which belong to this class, namely he proves that a space $S$ has the property $(E)$ if and only if it is normal. In 83 the author shows that in compact normal spaces the customary definition of dimension which is defined by means of finite coverings of a space coincides with one which is given by using essential mappings of a space on a finite dinensional simplex.

(M.T.)

49. Cn the probability distribution on a compact group. Yukiyosi KAWADA and Kiyosi ITO. [Proc. Phys.-Math. Sce. Japan Ser. 3, 22 (1940), 977-998.] Some properties on the probability distribution on a compact group, especially concerning the Markoff process with the help of characteristic matrices are given. In the appendix a mechod using Markoff process to get the mean value of a continuous function on a compact group is given.

Author.

50. An abstract integral. Sin-ichi IZUMI. [Proc. Imp. Acad. 16 (1940), 21-25.] The object of this paper is to define abstract integrals different from that of Riemann and Lebesgue.

51. The Markoff process with a stable distribution. Kôsaku YOSIDA. [Proc. Imp. Aced. 16 (1940), 43-48.] Let $R$ be a space and let $B(R)$ be a completely additive family of "measurable" subsets of $R$ and let $P(x, E)$ denote the transition probability that the point $x \in R$ is transferred, by a simple Markoff process, into the set $E \in B(R)$ after the elapse of unit time and the transition probability $P(n)(x, E)$ that the point $x$ is transferred into $E$ after the elapse of $n$ unit-times. Let a non-negative set function $\varphi(E)$ satisfy the conditions: (i) $\varphi(E)$ is completely additive for $E \in B(R)$ and $\varphi(R)=1$. (ii) The space $B(E)$, if metrised by the distance $d\left(E_{1}, E_{2}\right)=\varphi\left(E_{1}+E_{2}-E_{1} E_{2}\right)$, is complete and separable. (iii) $\left.\int \varphi d x\right) P(x, E)=\varphi E$ ) for any $E \in B(R)$. Let $(L)$ be the set of all real-valued measurable functions $f(x)$ which are $p$-integrable and $\|f\|=\int|f(x)| p(d x)$. The author proves the following theorems. (I) For any $f \in(L), f^{(m)}(x)=\int P^{(m)}(x, d y) f(y)$ $(m=1,2, \ldots)$ exists $q$-almost everywhere and belongs to $(L)$ in such a way that $\left\|f^{(m)}\right\| \leqq\|f\|$. For any $f \epsilon(L)$ and for any $m$, there corresponds $f(-m) \in(L)$, $\|f(-m)\| \leqq\|f\|$, such that $\left.\int \varphi(d x) f(x) P(m) x, E\right)=\int_{E^{\prime}} f(-m)(x) \uparrow(d x)$ for all $E \in B(R)$. (II) For any $f \in(L)$, there corresponds $\left.f^{(*)},\left(f^{(-*}\right) \in(L)\right)$ such that $\lim _{n \rightarrow \infty} \int \mid f^{*}(x)-\frac{1}{n} \sum_{m=1}^{n} f(m)(x) \rho(d x)=0$, $f^{*}(x)=\int P(x, d y) f^{*}(y)$, $\varphi$-almost everywhere, $\lim _{n \rightarrow \infty} \int\left|f(-*)(x)-\frac{1}{n} \sum_{n=1}^{n} f^{(-m)}(x)\right| \rho^{\prime}(d x)=0$, $\int q(d x) f^{(-*)}(x) P(x, E)=\int f(-*)(x) \varphi(d x)$ for any $E \in B(R)$.

52. Ergodic theorems and the Markoff process with a stable distribution. Shizuo KAKUTANI. [Proc. Imp. Acad. $16(1940), 49-54$.$] A Markoff process P(t, E)$ is called to have a stable distribution $q(E)$, if there exists a completely additive non-negative set function $\varphi(E)$ (with $\varphi(\Omega)=1$ ) defined for all Borel set $E \subset \Omega$ such that $\int_{\Omega} \varphi(d t) P(t, E)=\psi(E)$ for any Borel set $E \subset \Omega$. Let $x(t)$ bs any bounded Borel measurable function on $\Omega$ and $x_{n}(t)=\int_{\Omega} P(n)(t, d s) x(s)$. Let $x \rightarrow T(x)=y: y(E)=\int_{\Omega} x(d t) P(t, E)$ be 
a positive bounded linear operation of norm 1 which maps the Banach space $(\mathfrak{M})$ of all the completely additive real valued functions $x(E)(\|x\|=1)$ into itself and that

$$
x \rightarrow \bar{T}(x)=y: \quad y(t)=\int_{\Omega} P(t, d s) x(s)
$$

be a positive bounded linear operation of norm 1 which maps the Banach space $\left(M^{*}\right)$ of all the bounded Borel measurable real valued functions $x(t)(\|x\|=l$. u.b. of $x(t)$ on $\Omega$ ) into itself. The author proves the theorems. (I) Let $P(t, E)$ be a Markoff process with a stable distribution $\varphi(E)$. Then for any $x(t) \in M(\varphi)$, there exists an $x(t) \varepsilon M(\varphi)$ such that $\frac{1}{N} \sum_{n=1}^{N} x_{n}(t) \rightarrow \bar{x}(t)$ p-almost everywhere on $\Omega$. (II) Let $P(t, E)$ be a Markoff process with a stable distribution $\varphi(E)$. Then there exists for any $x(t) \in L(\varphi)$ an $\bar{x}(t) \in L(\varphi)$ such that $\int_{\Omega}\left|\frac{1}{N} \sum_{n=1}^{N} x_{n}(t)-\bar{x}(t)\right| \varphi(d t) \equiv\left\|\frac{1}{N} \sum_{n=1}^{N} \bar{T}^{n}(x)-\bar{x}\right\|$ where $x_{n}=\bar{T} n(x)$ and $L(\varphi)$ is the Banach space of all $\varphi$-integruble Borel measurable real valued functions $x(t)$ and $\|x\|=\int_{\Omega}|x(t)| \varphi(d t)$.

53. Über die ÜbŁrdeckungen von Zellenräumen, III. Atuo KOMATU. [Proc. Imp. Acad. 16 (1940), 55-58.] Es seien $K$ ein endlicher Zellenraum, $\Im$ die Fundamentalgruppe von $K$. $U$ sei eine Überdeckung von $K$ in bezug auf eine Koeffizientengruppe $\Im$. Dann bestimmt $U$ eine Darstellung, d.h. eine homomorphe Abbildung $g$ von $f$ in die Automorphismengruppe $\mathrm{r}$, die aus allen invertierbaren Automorphismen von $\Im$ auf sich besteht. Zwei U̇berceckungen $U_{1}$ und $U_{2}$ von $K$ in bezung auf $\Im$ heissen äquivalent, wenn $U_{1}$ und $U_{2}$ eine gleiche Darstellung $g$ von $\mathfrak{F}$ in $\Gamma$ bestimmen. $a^{r}$ sei eine Zelle von K. Ein Automorphismus $\gamma$ heisst Inzidenzautomorphismus bezüglich $a r$, wenn $\gamma$ bei $U$ zwischen $a^{r}$ und $a^{r+1}\left(a^{r+1}>a^{r}\right)$, oder zwischen $a^{r}$ und $a^{r-1}\left(a^{r}>a^{r-1}\right)$ definiert ist. Zwei äquivalente Überdeckungen $U_{1}$ und $U_{2}$ heissen benachbart, wenn ihre Automorphismen bis auf Inzidenzautomorphismen bezüglich eines $a^{r}$ identisch sind. Dann gilt: (I) Zwei äqivalente Überdeckungen $U_{1}$ und $U_{2}$ werden durch eine Folge von benachbarten Überdeckungen verbunden. (II) Zwei benachbarte Überdeckungen $U_{1}$ und $U_{2}$ von $K$ haben isomorphe Bettische Gruppen $B i\left(K, U_{1}\right)$ und $B i\left(K, U_{2}\right)$. (III) Äqivalente Überdeckungen haben isomorphe Bettische Gruppen. (From the author's introduction).

54. Über Authomorphismen der lokal kompakten abelschen Gruppen. Makoto ABE. [Prce. Imp. Acad. 16 (1940), 59-62.] Let $G$ be a locally compact abelian Group satisfying the second axiom of countability and $G^{*}$ its character group. The author proves that the ings of authomorphisms of $G$ and $G^{*}$ are topologically inverse-isomorphic to each octher. A corollrary and some examples are given.

55. Weak topology, bicompact set and the principle of duality. Shizuo KAKUTANI. [Proc. Imp. Acad. 16 (1940), 63-67.] Let $\subseteq$ be an abstract space and $\Omega$ the family of real -valued (bounded) function $f(x)$ defined on $\Omega$. The author introduces weak topologies on $\Omega$ and states that these weak popolgies are useful in the problems concerning the regularity of Banach space and in the theory of L. Pontrajagin and E.R. van Kampen concerning the duality of topclogical commuative groups. The detail of the proof will be published in another' paper.

56. Some theorems on abstractly-valued functions in an abstract space. Kiyonori KUNISAWA. [Proc. Imp. Acad. 14 (1940), 68-72.] Let $f(t)$ be an abstractily-valued function defined on $[0,1]$, whose range lies in a Banach space $\mathfrak{X}$. Let $L p(\mathfrak{X}) .(p \geqq 1)$ $\left(L^{1}(\mathfrak{X})=L(\mathfrak{X})\right)$ be the class of all functions $f(t)$ measurable in the sense of S. Bochner, 
such that $\int_{0}^{1}\|f(t)\| p d t<\infty . \quad L p(\mathfrak{x})(p \geqq 1)$ is a Banach space with $\|f\|=\left(\int_{0}^{1} \mid f(t) p d t\right)^{\frac{1}{p}}$ as its norm. The author proves the theorems: (I) In an arbitrary space $T$, let $\xi$ be a Borel family of subsets that includes $T$, and $\alpha(E)$ be a non-negative set function which is completely additive over $\xi$. If an abstractly-valued function $X(E)$, defined from $\xi$ to a Banabh space $\mathfrak{X}$, is weakly absolutely continuous (i.e. for each $\varphi$ in $\bar{x}$, the numerical function $\varphi X(E)$ is comrletely additive and absolutely continuous), then $X(E)$ is even strongly absolutely continuous (i.e. $X(E)$ is strongly completly additive, and for any $\varepsilon>0$, there exists a $\delta>0$ auch that $\|X(E)\|<\varepsilon$, whenever $\alpha(E)<\delta$ ). (II) If $\mathfrak{X}$ is locally weakly compact and if a sequence $\left.\left\{f_{n} t\right)\right\}$ of elements of $L(\mathfrak{X})$ is equi-integrable, then $\left\{f_{n}(t)\right\}$ contains a subsequence which converges weakly (as a sequence in $L(\mathfrak{X}))$ to an element $f(t) \in L(\mathfrak{X})$. (III) If $\mathfrak{X}$ is lccally weakly compact, then $L p(\mathfrak{X})(p>1)$ is also locally weakly compact. (IV). If $\mathfrak{x}$ is locally weakly compact, then $L p(\mathfrak{X})$ is weakly complete.

57. Sur un problème de M.E. Szilrajn. Kinjiro KUNUGUI. [Proc. Imp. Acad. 16 (1940), 743-778.] $M$ étant un ensemble quelconque situé dans le plan $O X Y$, désignons par $N x_{0}$ l'intersection de $M$ par la dnoite parallèle à l'axe $O Y$ menées par la point $\left(x_{0}, 0\right)$ et $\operatorname{par} \Delta(M)$ l'ensemble de tous les points $x_{0}$ de l'axe $O X$ tels que $M x_{0}$ soient des ensembles non vides et sont des ensembles $F_{\sigma}$. M.E. Szpilrajn a posé un problème. Soit $M$ un ensemble plan $G_{\delta}$ (plus genéralement un ensemble borelien) dont toutes les interseccions avec les droites parallèles à l'axe $O Y$ sont des ensembles fermés (plus généralement: des $F_{\sigma}$ ). La projection de $M$ sur l'axe $O X$ est-telle toujours un ensemble borelien?

Le but de ceite note est de démontrer le ćhéorème: Si $M$ est un ensemble borelien situé dans le plan, l'ensemble $\Delta(M)$ est toujours un complémentaire analytique.

58. On some properties of umbilical points of hypersurfaces. Yosio MUTÔ. [Proc. Imp. Acad. 16 (1940), 79-82.] Let us consider in an $(n+1)$-dimensional Riemannian space $V_{n+1}$ a hypersurface $V_{n}$. The author calls a point on the $V_{n}$ a perfectly umbilical point when there is a line of curvature passing through the point in each direction, and proves that the Weyl conformal curvature of a Riemannian space vanishes at a point if at this point in each direction there is a hyparsurface with the point as a perfectly umbilical point.

59. Conformally separable quadratic differential forms. Kentaro YANO. [Proc. Imp. Acad. $16(1940), 83-\varangle 6$.] Let $V_{n}$ be an $n$-dimensional Riemannian space whose first fundamental form is $d s^{2}=g^{\mu \nu} d u^{\mu} d u^{\nu}(\lambda, \mu, \nu, \ldots=1,2, \ldots, n)$. In this space, the equations $u^{a}=$ const. $(a, b, c, \ldots=1,2, \ldots, m<n)$ define a family of $(n-m)$-dimensional subspaces $V_{n-m}$ and the equations $u^{i}=$ const. $(i, j, k, \ldots=m+1, \ldots, n)$ a family of $m$-dimensional subspaces $V_{m}$ in $V_{n}$. It has been shown by Bompiani that the necessary and suffibient condition that the subspaces $V_{n-m}$ and the subspaces $V_{m}$ orthogonal to $V_{n-m}$ be both totally geodesic in $V_{n}$ is that the first fundamental form of $V_{n}$ may be written in the form: $d s^{2}=f_{a b}\left(u^{c}\right) d u^{a} d u^{b}+f_{j k}\left(u^{i}\right) d u^{j} d u^{k}$. Generalizing this theorem of Bompiani, the author shows that the necessary and sufficient condition that the subspaces $V_{n-m}$ and $V_{m}$ orthogonal to $V_{n-m}$ be both totally umbilical in $V_{n}(n-m \geqq 2$, $m \geqq 2$ ) is that the first fundamental form of $V_{n}$ has the following form: $d s^{\Sigma}=\rho\left(u^{\lambda}\right) f_{a b}\left(u^{c}\right) d u^{a} d u^{b}+\sigma\left(u_{\lambda}\right) f_{j k}\left(u^{i}\right) d u^{j} d u^{u}$. Such a quadratic form is said to be conformally separable. As an application of this theorem, the author obtains the theorem: If the first fundamental quadratic differential form of a Riemannian space of constant 
curvature is conformally separable into two components, then each component is also the first fundamental form of a Riemanian space of constant curvature. Author.

6o. An abstract integral. II. Shin-Ichi IZUMI. [Proc. Imp. Acad., 16 (1940), 8789.] The object of this paper is to define Riemann and Lebesgue integrals of functions whose values belong to a very general space. For this purpose the author defines new definitions of Riemann and Lebesgue integrals. More generally, the definitions of Riemann-Stieltjes and Lebesgue-Stieltjes integrals are given which are free from the notions of paritition, least upper bound and greatest lower bound.

61. Grundlage der Geometrie in der Mannigfaltigkeit der Kurvenelemente. Hitoshi HOMBU. [Prcc. Imp. Acad. $16(1940)$, 9)-96.] Let $x^{i}(t)$ be a parametrized curve in an $n$-dimensional manifcld. The $x_{d x a}^{i}, \frac{d x^{i}}{d t}, \ldots, \frac{d^{\nu} x^{i}}{d t^{\nu}}$ are called linear element of the $v$-th order and $x^{i}, \frac{d x^{a}}{d x^{n}}, \ldots, \frac{d^{\nu} x^{a}}{d x^{2}}$ curve elements of the $v$-th order. The author investigates the conditions that the functions and Pfaff forms of these linear elements are expressible in terms of the curve elements. These considerations are fundamental in the higher order geometry.

(M.T.)

62. Die Geometrue des Integrals $\int F\left(x, x^{(1)}, \ldots x^{(m)}\right) d t$. Hitoshi HOMBU. [Proc. Imp. Acad., 16 (1940), 97-103.] In the light of the former work (see the above review), the author develops the geometry of the integral $\int F\left(x, x^{(1)}, \ldots, x(m)\right) d t$. The strong use of the author's theory of paths has been made.

63. Über die Geometrie des Systems der partiellen Differentialgleichungen dritter Ordnung. Shisanji HOKARI. [Proc. Imp. Acad., 16 (1940), 104-108.] The author considers a system of partial differential equations of the third order

$$
\frac{\partial^{3} x^{i}}{\partial u^{\alpha} \partial u^{\beta} \partial u^{\gamma}}+H_{\alpha}^{i} r\left(u^{\lambda}, x^{j}, \frac{\partial x^{j}}{\partial u^{\lambda}}, \frac{\partial^{2} x^{j}}{\partial u^{\lambda} \partial u^{\mu}}\right)=0
$$

defining $K$-dimensional surfaces in an $n$-dimensional manifold, and develops the intrinsic (in the sense of $\mathrm{E}$. Bortolotit) theory of these partial differential equations. The covariant derivative on the integral surface of a mixed tensor via being defined by

$$
\delta v^{i \alpha}=d v^{i \alpha}+\Gamma_{j \gamma}^{i} v^{j \alpha} d u r+G_{\beta \gamma}^{\alpha} v^{i \beta} d u^{r},
$$

the author determines intrinsically $\Gamma_{j r}^{i}$ and $G_{\beta r}^{\alpha}$ in terms of $H_{\alpha \beta r}^{i}$.

64. On the strong law of large numbers. Tatuo KAWATA. [Proc. Imp. Acad., I6 (1940), 109-112.] Let $X_{1}, X_{2}, \ldots, X_{n}$ be a sequence of independent chance variables and let the expectation of $X_{n}, E\left(X_{n}\right)$ be 0 . If $\frac{S_{n}}{n}=\frac{X_{1}+X_{2}+\cdots+X_{n}}{n}$ converges to zero with probability 1, we say that the sequence $\left\{X_{n}\right\}$ obeys to the strong law of large numbers. The auchor proves the theorems. (I) For any positive $\varepsilon>0$, let $P_{r}\left(\varepsilon>\frac{S_{n}}{n}>-\varepsilon\right)>1-\hat{o}_{n}(\varepsilon), \hat{o}_{n}(\varepsilon) \rightarrow 0$ as $n \rightarrow \infty$ and suppose that for any $\varepsilon>0$, $\sum_{k=1}^{\infty} \hat{o}_{2} k(\varepsilon)<\infty$. Then the sequence $X_{n}$ converges to zero with probability 1 . (II) Let $E\left(X_{n}^{2}\right)=b_{n}$. If $\sum_{n=1}^{\infty} \frac{b_{n}}{n^{2}}<\infty$, the sequence $\left\{X_{n}\right\}$ obeys to the strong low of large numbers. 
65. Über die Zerlegung der Charaktere der alternierenden Gruppe. Kôiti KONDÒ. [Proc. Imp. Acad., 16 (1940), 131-135.] From the well-known relationship between the characters of the alternating groups $\mathfrak{A}_{n}$ and those of the symmetric groups $\mathfrak{S}_{n}$, and the further relationship between the characters of $\subseteq_{n}$ and $\subseteq_{n-1}$, the author deduces the relationships between the characters of $\mathfrak{I}_{n}$ and $\mathfrak{A}_{n-1}$.

Author.

66. On the theory of almost periodic functions in a group. Shokichi IYANAGA and Kunihiko KODAIRA. [Prcc. Imp. Acad., 16(1940), 136-140.] The authors propose a modified defi ition of the almost periodic functions on a group on the sense of the $v$. Neumann and give a simple proof of the existence of the mean value for such functions.

Authors.

67. A remark on the non-vanishing of almost periodic functions. Tatsuo KAWATA. [Proc. Imp. Acad., $16(1940), 157-160$.$] The author proves that: (i) f(x)$ is said to be $S^{2}$, a.p., if there exists a sequence of trigonometrical polynomials $f_{n}(x)$ such that $\lim _{n \rightarrow \infty} D\left(f-f_{n}\right)=0$, where $D(f)=\underset{-\infty<b<<<}{\text { u.b. }}\left\{\int_{x}^{x+1}\left\{\left.f(x)\right|^{2} d x\right\}^{\frac{1}{2}}\right.$. Let $f(x)$ be $S^{2}$ a.p. and its Fourier exponents greater than some number $A$ have gaps tending to infinity and $g(z)=g(x+i y)$ be a function analytic in $c<x<d, o<y<r$ and continuous in $c \leqq x \leqq d$, $o \leqq y \leqq r$, and $f(x)=g(x)$ almost everywhere in some interval in $(c, d)$, then $f(x)=g(x)$ almost everywhere in $(c, d)$. (ii) Let $f(x)$ be $S^{2}$ a.p. and its Fourier series be $\sum a_{n} e^{i \lambda_{n} x}$. Let there exist an $N$ such that $A(u)=\sum_{-u-1 / 2<\lambda_{k}<-u+1}\left|\alpha_{k}\right|$ be finite for each $u(\geqq N)$ and $A(u)=O\left(e^{-\theta}(u)\right)$, where $\int_{1}^{\infty} \frac{\theta(x)}{x^{2}} d x=\infty$. Further let $g(z)=g(x+i y)$ be a function analytic in $c<x<d, o<y<r$ and continuous in $c \leqq x \leqq d, o \leqq y<r$. Then if $f(x)=g(x)$ almost everywhere in the interval $(c, d)$, then it holds almost everywhere in $(c, d)$.

68. On affine geometry of abelian group. Kôshichi TOYODA. [Proc. Imp. Acad., $16(1940,161-164$.$] Let G$ be a set of elements $a, b, \ldots$ which satisfy some axioms on the product $a, b$. The author constitutes an affine geometry from $G$ under some conditions.

69. Über die Differenzierbarkeit der einparametrigen Untergruppe Liescher Gruppen. Kunihiko KODAIRA. [Proc. Imp. Acad., 16 (1940), 165-166.] A simple proof of the known theorem that every continuous one-parameter subgroup of a Lie group is differentiable with resrect to the parameter.

70. Über zusammenhängende kompakte abelsche Gruppen. Kunihiko KODAIRA und Makoto ABE: [Proc. Imp. Acad., 16 (1940), 167-172.] Following theorems are proved. 1) Every connected compact abelian group $G$ is topologically isomorphic to its onedimensional Betti-group $B_{k}^{1}(G)$ with the additive group $\AA$ of the real numbers mod. 1 as coefficient group. 2) Let $G^{\prime}$ bs another group of the same nature. In every homotopy class of the continuous mapping of $G$ in $G^{\prime}$ there is exactly one continuous homomorphism of $G$ in $G^{\prime}$.

71. Sur quelques propriêté conformes de $V_{i}$ dans $V_{m}$ dans $V_{n}$. Kentaro YANO. [Proc. Imp. Acad., 16 (1940), 173-177.] Let $V_{m}$ be a subspace of a Riemannian space $V_{n}$ and $V_{l}$ be a Riemannian subspace of the subspace $V_{m}$. Then we can consider three Euler-Schouten tensors $H_{a b}^{\cdot i}$ of $V_{l}$ with respect to $V_{m}, H_{a b}^{\cdot{ }^{\lambda}}$ of $V_{l}$ wich respect to $V_{n}$ and $H_{j k}{ }^{\lambda}$ of $V_{m}$ with respect to $V_{n}$. Between these tensors there are well known relations $H_{a b}^{\cdot{ }^{\lambda}}=H_{j k}{ }^{\lambda} B_{a}^{\cdot j} B_{b}^{k}+B_{i}^{{ }^{\lambda}} H_{a b}{ }^{i}$. Generalizing these formulae, the author obtains 


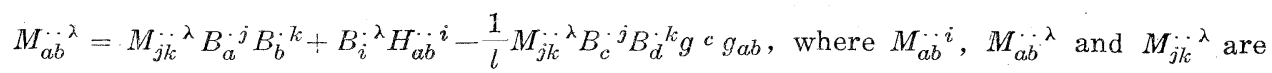
conformally invariant tensors derived from $H_{a \dot{b}}{ }^{i}, H_{a \dot{b}}{ }^{\lambda}$ and $H_{j k}{ }^{\lambda}$ respectively and the identical vanishing of which represents the property that the subspace is totally umbilical in the containing space. From the latter relation, the author obtains the following theorems. If $V_{l}$ is totally umbilical in $V_{m}$ and $V_{m}$ is also totally umbilical in $V_{n}$, then $V_{l}$ is totally umbilical in $V_{n}$. If $V_{l}$ is totally umbilical in $V_{n}$, then $V_{l}$ is totally umbilical also in $V_{m}$. The intersection $V_{n-2}$ of two totally umbilical hypersurfaces $V_{n-1}$ and $* V_{n-1}$ of $V_{n}$ is also totally umbilical.

Author.

72. Concircular geometry I. Concircular transformations. Kentuo YANO. [Proc. Imp. Acad., 16 (1940), 195-200.] The author defines the geodesic circles in a Riemannian space $V_{n}$ as the curves whose first curvature is constant and the second curvature vanishes identically. Then the differential equations of the circles are given by $\frac{\delta^{2} u^{\lambda}}{\delta s^{3}}+\frac{d u^{\lambda}}{d s} g_{\lambda \nu} \frac{\delta^{2} u^{\lambda}}{\delta s^{2}} \frac{\bar{\delta}^{2} u^{\nu}}{\delta s^{2}}=0$. By a general conformal transformation $\overline{g_{\mu \nu}}=\delta^{2} g_{\mu \nu}$ of

Riemannian spaces, the geodesic circles are not in general transformed into geodesic circles. The necessary and sufficient condition that any geodesic circle be transformed into a geodesic circle by a conformal transformation $\bar{g}_{\mu \nu}=\delta^{2} g_{\mu \nu}$ is that the function $\rho$ satisfies the partial differential equations

$$
\rho_{\mu \nu} \equiv \rho_{\mu, \nu}-\rho_{\lambda}\left\{\begin{array}{c}
\lambda \\
\mu \nu
\end{array}\right\}-\rho_{\mu} \rho_{\nu}+\frac{1}{2} g^{\alpha \beta} \rho_{\alpha} \rho_{\beta} g_{\mu \nu}=\phi g_{\mu \nu} \text {, where } \rho_{\mu}=\frac{\partial \log \rho}{\partial u_{\mu}} \text { and } \rho_{\mu, \nu}=\frac{\partial \rho_{\mu}}{\partial \mu^{\nu}} \text {. }
$$

A conformal transformation with $p$ satisfying the last relation changing a geodesic circle into a geodesic circle, the author calls it concircular transformation and calls concircular geometry the geometry in which one concerns only with the concircular transformations and the spaces admitting such transformations. After having explained the idea of the concircular geometry, the author proves the following fundamental theorems: I. The necessary and sufficient condition that a Riemannian space $V_{n}$ may be reduced to a Euclidean space by a suitable concircular transformation is that the

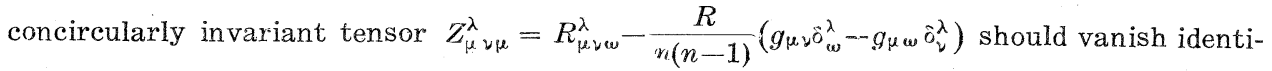
cally. II. A space of constant curvature is transformed into a space of constant curvature by a concircular transformation. III. An Einstein space is transformed into an Einstein srace by a concircular transformation.

Author.

73. On the division of a probability law. Tatsuo KAWATA. [Proc. Imp. Acad., $16(1940), 249$ 254.] If a random variable $X$ is represented as a sum $X_{1}+X_{2}$ of independent variables $X_{1}$ and $X_{2}$; in other words, if the characteristic function of $X$, $f(t)=\int_{-\infty}^{\infty} e^{i t x} d \sigma(x), \sigma(x)$ being the distribution function of $X$, is represented as a product $f_{1}(t), f_{2}(t)$ of characteristic functions $f_{1}(t)$ and $f_{2}(t)$ of $X_{1}$ and $X_{2}$ respectively, $X$ is said to be divisible by $X_{1}$ or $X_{2}$. The division of $X$ by some random variable is not necessarily determined uniquely. The purpose of the paper is to discuss the unicity of divisibility in terms of a distribution function of $X$.

74. A relation between the theories of Fossrier series and Fourier transforms. Tatsuo KAWATA. [Proc. Imr. Acad., 15(1940), 255-261.] Le' $f(x)$ be defined in $(-\infty, \infty)$ and belong to some class $L_{p}(p \geqq 1)$. If there exists a function $F(t)$ such that $\lim _{A \rightarrow \infty} \int_{-\infty}^{\infty} F(t)-\left.\frac{1}{\sqrt{2 \pi}} \int_{-A}^{A} f(x) e^{-i t x} d x\right|^{q} d t=0$, then $F^{\prime}(t)$ is called the Fourier transform of $f(x)$ in $L_{i q}$. The author proves the following theorems. (i) Suppose that $f(x) \in L_{p}(-\infty, \infty)$ 